\title{
IDH1 gene mutation activates Smad signaling molecules to regulate the expression levels of cell cycle and biological rhythm genes in human glioma U87-MG cells
}

\author{
YONGYING GAO ${ }^{1,2^{*}}$, YANWEI WU ${ }^{1 *}$, NINGMEI ZHANG ${ }^{3 *}$, HONGMEI YUAN ${ }^{4}$, FEI WANG $^{5}$, \\ HUI XU ${ }^{1}$, JIAXIANG YU ${ }^{1}$, JIE MA ${ }^{1}$, SHAOZHANG HOU ${ }^{1}$ and XIANGMEI CAO ${ }^{1}$ \\ ${ }^{1}$ Department of Pathology, School of Basic Medicine, Ningxia Medical University, Yinchuan, Ningxia 750004; \\ ${ }^{2}$ Department of Neurology, Ningxia Hui Autonomous Region People's Hospital, Yinchuan, Ningxia 750021; \\ ${ }^{3}$ Department of Pathology, Tumor Hospital, General Hospital of Ningxia Medical University, Yinchuan, Ningxia 750004; \\ ${ }^{4}$ Functional Department, Ningxia Hui Autonomous Region People's Hospital, Yinchuan, Ningxia 750021; \\ ${ }^{5}$ Department of Pathology, The First People's Hospital of Yinchuan, Yinchuan, Ningxia 750001, P.R. China
}

Received September 4, 2020; Accepted February 9, 2021

DOI: $10.3892 / \mathrm{mmr} .2021 .11993$

\begin{abstract}
Isocitrate dehydrogenasel (IDH1) mutation is the most important genetic change in glioma. The most common IDH1 mutation results in the amino acid substitution of arginine 132 (Arg/R132), which is located at the active site of the enzyme. IDH1 Arg132His (R132H) mutation can reduce the proliferative rate of glioma cells. Numerous diseases follow circadian rhythms, and there is growing evidence that circadian disruption may be a risk factor for cancer in humans. Dysregulation of the circadian clock serves an important role in the development of malignant tumors, including glioma. Brain-Muscle Arnt-Like protein 1 (BMAL1) and Circadian Locomotor Output Cycles Kaput (CLOCK) are the main biological rhythm genes. The present study aimed to further study whether there is an association between IDH1 R132H mutation and biological rhythm in glioma, and whether this affects the occurrence of glioma. The Cancer Genome Atlas (TCGA) database was used to detect the expression levels of the biological rhythm genes BMAL1 and CLOCK in various types of tumor. Additionally, U87-MG cells were infected with wild-type and mutant IDH1 lentiviruses. Colony formation experiments were used to detect cell proliferation in each group, cell cycle distribution was detected by flow cytometry
\end{abstract}

Correspondence to: Professor Xiangmei Cao or Professor Shaozhang Hou, Department of Pathology, School of Basic Medicine, Ningxia Medical University, 1160 Shengli Road, Yinchuan, Ningxia 750004, P.R. China

E-mail: caoxm.nxmu@163.com

E-mail: houshzh@nxmu.edu.cn

${ }^{*}$ Contributed equally

Key words: isocitrate dehydrogenase 1 mutation, biorhythm, glioma, cyclins, Smad and western blotting was used to detect the expression levels of wild-type and mutant IDH1, cyclins, biological rhythm genes and Smad signaling pathway-associated genes in U87-MG cells. TCGA database results suggested that BMAL1 and CLOCK were abnormally expressed in glioma. Cells were successfully infected with wild-type and mutant IDH1 lentiviruses. Colony formation assay revealed decreased cell proliferation in the IDH1 R132H mutant group. The cell cycle distribution detected by flow cytometry indicated that IDH1 gene mutation increased the $G_{1}$ phase ratio and decreased the $S$ phase ratio in U87-MG cells. The western blotting results demonstrated that IDH1 R132H mutation decreased the expression levels of the S phase-associated proteins Cyclin A and CDK2, and increased the expression levels of the $\mathrm{G}_{1}$ phase-associated proteins Cyclin D3 and CDK4, but did not significantly change the expression levels of the $\mathrm{G}_{2} / \mathrm{M}$ phase-associated protein Cyclin B1. The expression levels of the positive and negative rhythm regulation genes BMAL1, CLOCK, period (PER s (PER1, 2 and 3) and cryptochrom (CRY)s (CRY1 and 2) were significantly decreased, those of the Smad signaling pathway-associated genes Smad2, Smad3 and Smad2-3 were decreased, and those of phosphorylated (p)-Smad2, p-Smad3 and Smad4 were increased. Therefore, the present results suggested that the IDH1 R132H mutation may alter the cell cycle and biological rhythm genes in U87-MG cells through the TGF- $\beta /$ Smad signaling pathway.

\section{Introduction}

Isocitrate dehydrogenase (IDH) genes are mutated in multiple types of tumor, including glioma, chondrogenic tumors, leukemia and other bone marrow proliferative tumors. In glioma and leukemia, IDH1 and IDH2 mutations occur in $>70 \%$ of low-grade tumors (level II and III) $(1,2)$. IDH mutation is the most important genetic change in glioma. The mutation is located at the isocitric acid binding site (Arg/R132 of IDH1 and R172 of IDH2) of a single amino acid $(3,4)$. IDH1/2 mutations are common in World Health Organization (WHO) 
grade II and III gliomas and secondary glioblastoma (GBM; 70-80\%), while primary GBM (WHO grade IV) IDH1/2 mutations are rare $(<5 \%)(5,6)$. IDH1 mutations account for $>90 \%$ of glioma IDH1/2 mutations (7). IDH1 Arg132His (R132H) mutation can affect the proliferation of glioma cells, which is slower than the corresponding wild-type IDH1 cells $(8,9)$. Clinical studies $(10,11)$ have shown that mutations in IDH1 were found to be associated with younger age, secondary GBMs (grade IV tumors that arise from biopsy-proven lower-grade predecessors), and increased overall survival (OS) (12). Further studies $(13,14)$ have revealed that IDH1/2 mutations as good prognostic markers are universally present in grade II and III glioma and secondary glioblastoma, and serve an important role in the occurrence, development and evolution of glioma (15). Therefore, studying the role of the IDH1 R132H mutation in the occurrence of glioma may provide new ideas for clinical treatment.

There is growing evidence that dysregulation of the circadian clock serves an important role in the development of malignant tumors, including glioma $(16,17)$. Circadian timing is a basic biological process that affects most aspects of eukaryotic and prokaryotic physiology. Circadian dysrhythmia may lead to an increased risk of cancer, as well as affect the response of patients with cancer to treatment (18). In a circadian rhythm, the oscillator is coordinated by a set of interlocked transcription-translation feedback loops. Brain-Muscle Arnt-Like protein 1 (BMAL1) and Circadian Locomotor Output Cycles Kaput (CLOCK) are the main biological rhythm genes (19). CLOCK and BMAL1 heterogeneous dimers combined with Period (PER) and Cryptochrom (CRY) proteins in E-box device drive rhythmic transcription (20-22). The PER and CRY proteins inhibit CLOCK-BMAL1 complexes, which inhibit PER and CRY protein degradation after release (23-25). Upon epigenetic modification and increase post-translational modifications, the core CLOCK proteins in the suprachiasmatic nucleus can maintain peripheral CLOCK oscillation and rhythmic expression (26), and the downstream targets are similar to those in the suprachiasmatic nucleus; the molecular biological clock of the peripheral tissues and organs of the body is also composed of a transcription-translation feedback loop regulated by clock genes (27-32). Disruption of circadian rhythms may negatively affect normal cellular function and may lead to an increased incidence of multiple types of cancer, such as colorectal cancer (33), breast cancer (34), prostate cancer (35), pancreatic cancer (36), osteosarcoma (37) and others $(38,39)$. Therefore, it is very important to study the role of the circadian clock in the development and progression of cancer. Mutations in circadian clock components can increase the proliferation rate of cells through the general dysregulation of the cell cycle, thereby causing the cell to become cancerous (40). Several studies have revealed an interaction between the biological clock and the cell cycle. For example, a previous study has indicated that light-induced phase shifts in mouse behavior lead to corresponding changes in the time of intestinal cell proliferation (41). Another study has suggested that clock genes are involved in the regulation of important cell cycle checkpoints (42).

Understanding the mechanism of circadian clock changes in tumors is of great importance for the improvement of tumor therapy. However, to the best of our knowledge, the mechanism of biorhythm change in glioma with IDH1 R132H mutation has not been previously reported. In the present study, the
IDH1 R132H mutant gene was introduced into human glioma U87-MG cells to observe the effect of IDH1 on biological rhythm genes and to analyze its relevant mechanism, laying a theoretical foundation for the study of the effect of biological rhythm on the biological function of malignant tumors.

\section{Materials and methods}

Cell line and culture. The full name of the cell line used is U87-MG human brain astroblastoma, which is a GBM of unknown origin. Cells were cultured in Minimum Essential Medium (MEM; Shanghai Zhongqiao Xinzhou Biotechnology Co., Ltd.) containing 10\% fetal bovine serum (FBS; HyClone; Cytiva) at $37^{\circ} \mathrm{C}$ with $5 \% \mathrm{CO}_{2}$. The cell line was purchased from Shanghai Zhongqiao Xinzhou Biotechnology Co., Ltd.

Reagents and instruments. pLVX-IDH1-mCMV-ZsGreenPGK-Puro and pLVX-IDH1(MUT)-mCMV-ZsGreen-PG K-Puro lentiviruses were purchased from Beijing Xibei Hongcheng Biotechnology Co., Ltd. (http://www.xbhcbio. $\mathrm{com} /)$. Trypsin $(0.25 \%)$-EDTA was purchased from Invitrogen (Thermo Fisher Scientific, Inc.) and $0.45-\mu \mathrm{m}$ PVDF membranes and a chemiluminescence kit (Immobilon Western Chemiluminescent HRP Substrate,eCl@ss cat. no. 42029053) were purchased from EMD Millipore. A total protein extraction kit (Whole protein extraction kit, cat. no. KGP250), a BCA assay protein content detection kit (BCA Protein Quantitation Assay, cat. no. KGPBCA), an SDS-PAGE gel preparation kit (KGI SDS-PAGE Gel Preparation kit, cat. no. KGP113) and a flow cytometry cell cycle analysis kit (Cell Cycle Detection kit, cat. no. KGA511) were purchased from Jiangsu Kaiji Biotechnology Co., Ltd. (http://keygentec.com.cn/index.php?cid=1). The BSA (BSA-V, cat. no. A8020) used to dilute the antibody was purchased from Beijing Solarbio Science \& Technology Co., Ltd. Mouse anti-IDH1 (R132H; cat. no. SAB4200548) was purchased from Sigma-Aldrich (Merck KGaA). Rabbit anti-IDH1 (cat. no. 8137S), rabbit anti-BMAL1 (cat. no. 14020S), rabbit anti-CLOCK (cat. no. 5157S), rabbit anti-phosphorylated (p)-Smad2 (cat. no. 18338S), rabbit anti-p-Smad3 (cat. no. 9520S), rabbit anti-Smad2 (cat. no. 5339S), rabbit anti-Smad2-3 (cat. no. 86855S), rabbit anti-Smad3 (cat. no. 9513S), rabbit anti-Smad4 (cat. no. 46535S), mouse anti- $\beta$-actin (cat. no. 3700S) and mouse anti-GAPDH (cat. no. 51332S) primary antibodies, as well as HRP-labelled goat anti-rabbit IgG (cat. no. 7074S) and goat anti-mouse IgG (cat. no. 7076S) secondary antibodies, were purchased from Cell Signaling Technology, Inc. Rabbit anti-Cyclin A (cat. no. AF0142), rabbit anti-CyclinB1 (cat. no. DF6786), rabbit anti-CyclinD3 (cat. no. DF6229), rabbit anti-CDK2 (cat. no. AF6237), rabbit anti-CDK4 (cat. no. DF6102), rabbit anti-P18 (cat. no. AF0620), rabbit anti-P21 (cat. no. AF6290), rabbit anti-P27 (cat. no. AF6324), rabbit anti-PER1 (cat. no. DF9080), rabbit anti-PER2 (cat. no. DF12304), rabbit anti-PER3 (cat. no. DF7349), rabbit anti-Cry1 (cat. no. DF8932) and rabbit anti-Cry2 (cat. no. DF12919) primary antibodies were purchased from Affinity Biosciences. An IX73 inverted fluorescence microscope was purchased from Olympus Corporation, and an Amersham Imager 600 gel imaging analysis system was purchased from General Electric. 
The cancer genome atlas (TCGA) database analysis. BMAL1 and CLOCK expression data of all types of tumor analyzed in the present study were derived from TCGA database (https://portal.gdc.cancer.gov/) and the online analysis software GEPIA2 (http://gepia2.cancer-pku.cn/\#index).

U87-MG human glioma cell culture and lentiviral infection. Construction of recombinant lentiviral vector: $1 \mu \mathrm{g}$ Plasmid and pLVX/IDH1, $1 \mu \mathrm{l}$ EcoRI, $1 \mu \mathrm{l}$ BamHI, $2 \mu \mathrm{l}$ pLVX, $6 \mu \mathrm{l}$ target gene, $1 \mu 1$ 10X DNA Ligase Buffer and $1 \mu 1$ T4 DNA Ligase, were used to construct lentiviral vectors. The 239T cell line (Beijing Xibei Hongcheng Biotechnology Co., Ltd.) was used for virus purification. After culturing for 12 to $16 \mathrm{~h}$, the medium was discarded (500 $\mu$ l HET Buffer $\mathrm{A}+10 \mu \mathrm{l}$ recombinant lentiviral vector $+15 \mu l$ lentiviral packaging vector + HET Buffer B $50 \mu l+$ $\mathrm{ddH}_{2} \mathrm{O} 425 \mu \mathrm{l}$ ), and replaced with $10 \mathrm{ml}$ fresh complete medium solution (DMEM + 10\% FBS + P/S). The cells were placed in a $37^{\circ} \mathrm{C}, 5 \% \mathrm{CO}_{2}$ incubator for $48 \mathrm{~h}$, the supernatant was collected after centrifugation at $500 \mathrm{xg}$ and room temperature for $5 \mathrm{~min}$ and the cell debris was discarded. The supernatant was filtered with a $45 \mu \mathrm{m}$ PVDF filter into a $50 \mathrm{ml}$ round bottom centrifuge tube. This was centrifugated at a high speed of $6,000 \mathrm{xg}$ at $4^{\circ} \mathrm{C}$ for $2 \mathrm{~h}$, and purified recombinant lentivirus was obtained after centrifugation. Lentivirus can be introduced into U87-MG cells after $48 \mathrm{~h}$ of infection with MOI value (20:1) (43). U87-MG cells were inoculated in MEM containing $10 \%$ FBS at a density of $2 \times 10^{5}$ cells $/ 100-\mathrm{mm}$ culture dish, incubated at $37^{\circ} \mathrm{C}$ and then subjected to lentivirus infection 6-8 $\mathrm{h}$ after becoming adherent. The titre of the treatment and control lentiviruses was set to an MOI of 20:1, and MEM with 2\% FBS was used to prepare viral titre gradient solutions for subsequent experiments. The experimental groups were the IDH1 wild-type (WT) and the IDH1 $\mathrm{R} 132 \mathrm{H}$ mutant (MUT) groups, and the corresponding lentiviral vectors were pLVX-IDH1-mCMV-ZsGreen-PGK-Puro and pLVX-IDH1(MUT)-mCMV-ZsGreen-PGK-Puro, respectively. Subsequent experiments were performed $72 \mathrm{~h}$ after lentiviral infection.

Western blot analysis. Following lentiviral transfection, U87-MG cells in each group were collected for extraction of total protein using the aforementioned whole protein extraction kit. The BCA assay kit was used to quantify the protein, and equal amounts of protein $(40 \mu \mathrm{g} / 10 \mu \mathrm{l})$ were separated via $8 \%$ SDS-PAGE. Subsequently, the proteins were transferred to PVDF membranes, which were blocked at room temperature in $5 \%$ skimmed milk for $1 \mathrm{~h}$ and then incubated at $4{ }^{\circ} \mathrm{C}$ overnight with the following primary antibodies diluted with 3\% BSA: Anti-IDH1 (R132H; 1:1,000), anti-IDH1 (1:1,000), anti-GAPDH $(1: 2,000)$, anti- $\beta$-actin $(1: 2,000)$, anti-BMAL1 $(1: 1,000)$, anti-CLOCK (1:1,000), anti-PER1/2/3 (1:1,000), anti-CRY1/2 $(1: 1,000)$, anti-CyclinA $(1: 1,000)$, anti-CyclinB1 $(1: 1,000)$, anti-Cyclin D3 $(1: 1,000)$, anti-CDK2 $(1: 1,000)$, anti-CDK4 (1:1,000), anti-P18 (1:1,000), anti-P21 (1:1,000), anti-P27 (1:1,000), anti-p-Smad2 (1:1,000), anti-p-Smad3 (1:1,000), anti-Smad2 $(1: 1,000)$, anti-Smad2-3 $(1: 1,000)$, anti-Smad3 $(1: 1,000)$ and anti-Smad4 $(1: 1,000)$. The membranes were incubated with secondary antibodies (HRP-conjugated goat anti-rabbit IgG and goat anti-rat IgG; both 1:2,000) at room temperature for $1 \mathrm{~h}$. The chemiluminescence reagent was used for color development for $1 \mathrm{~min}$, and the membranes were exposed for greyscale measurement. The protein expression level was semi-quantified using Image Pro Plus 6.0 (Media Cybernetics, Inc.).

Colony formation experiments. After seeding 1,000 lentivirus-infected U87-MG cells into $100-\mathrm{mm}$ culture dishes, the cells were divided into the WT and MUT groups. After 5 days of culture, the medium was changed once, and whether the cells formed clumps or sheets was observed under a 10x light microscope. The medium was then discarded. The cells were fixed in $4 \%$ paraformaldehyde at room temperature for $20 \mathrm{~min}$. Methylene violet solution (4 ml) was added to each dish, and the cells were stained at room temperature for $30 \mathrm{~min}$ before rinsing with pure water. After drying at room temperature, images were captured, and the colonies, which are clumps or flakes formed by cells, were counted using Image-Pro Plus 6.0 (Media Cybernetics, Inc.) for statistical analysis.

Cell cycle distribution detection by flow cytometry. A cell cycle analysis kit (Cell Cycle Detection kit, cat. no. KGA511) was used for cell cycle detection. The cells in each group were digested with trypsin at $37^{\circ} \mathrm{C}$ for $1 \mathrm{~min}$, collected by centrifugation at $1,500 \mathrm{x}$ g for $5 \mathrm{~min}$ at normal temperature and rinsed with PBS to form a cell suspension. The concentration was adjusted to $1 \times 10^{6}$ cells $/ \mathrm{ml}$. After removing the supernatant by centrifugation at $1,500 \mathrm{x}$ g for $5 \mathrm{~min}$ at normal temperature, $500 \mu \mathrm{l}$ precooled $75 \%$ ethanol was used to fix the cells overnight at $4^{\circ} \mathrm{C}$. The fixation solution was removed by centrifugation at $1,500 \mathrm{x}$ for $5 \mathrm{~min}$ at $4^{\circ} \mathrm{C}, 100 \mu 1$ RNase A was added and the cells were incubated in a water bath at $37^{\circ} \mathrm{C}$ for $30 \mathrm{~min}$. A $400 \mu \mathrm{m}$ mesh screen was used for filtration. Then, $400 \mu \mathrm{l}$ PI dye was added and the solution was gently mixed and incubated at $4^{\circ} \mathrm{C}$ for $1 \mathrm{~h}$ in the dark. Analysis was performed using a flow cytometer (FACSCalibur, BD Biosciences; FlowJo, Version 10, FlowJo LLC).

Statistical analysis. SPSS 21.0 (IBM Corp.) statistical software was used for statistical analysis. The data are expressed as the mean $\pm \mathrm{SD}$. Each independent experiment was repeated three times. Independent sample t-test was used to analyze differences between two groups of continuous data. One-way ANOVA was used for the comparison among multiple groups followed by Tukey's post hoc test. $\mathrm{P}<0.05$ was considered to indicate a statistically significant difference.

\section{Results}

Biological rhythm genes are abnormally expressed in glioma. BMAL1 and CLOCK expression was analyzed in TCGA database. The expression levels of BMAL1 and CLOCK appeared to differ among the 33 types of tumor. BMAL1 expression in acute myeloid leukemia was higher than that in normal tissues (Fig. 1A). By contrast, BMAL1 expression was lower in diffuse large adrenocortical carcinoma (ACC), cervical squamous cell carcinoma, endocervical adenocarcinoma (CESC), colon adenocarcinoma (COAD), lung adenocarcinoma, lung squamous cell carcinoma, ovarian serous cystadenocarcinoma, prostate adenocarcinoma, rectum adenocarcinoma, skin cutaneous melanoma, testicular germ cell tumors (TGCT), uterine corpus endometrial carcinoma and uterine carcinosarcoma compared with that in normal tissues (Fig. 1A). Additionally, BMAL1 expression in brain lower grade glioma (LGG) tissues was lower than that in 

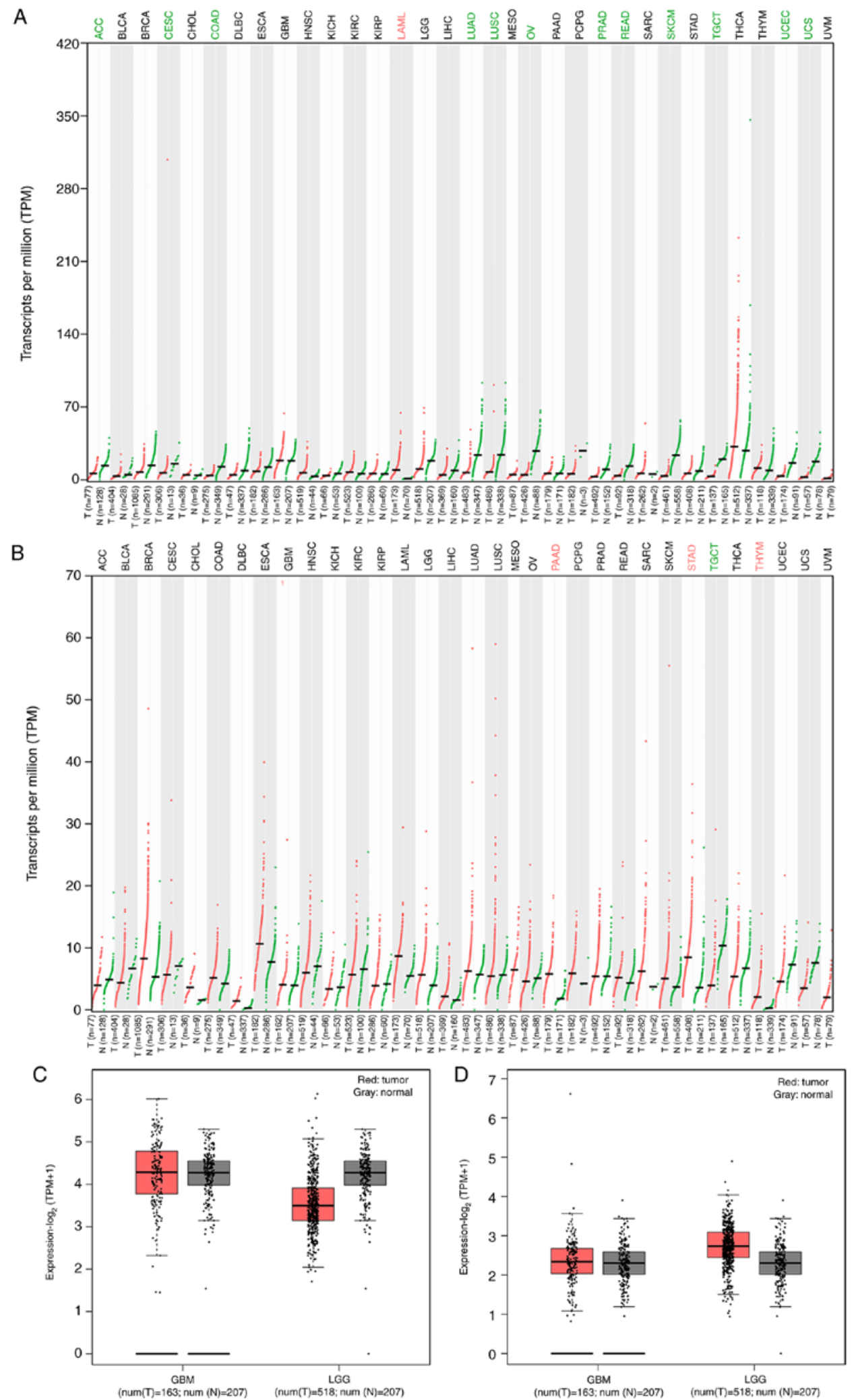

Figure 1. Expression of BMAL1 and CLOCK in different tumors in TCGA database. (A) BMAL1 and (B) CLOCK expression in 33 types of tumor from The Cancer Genome Atlas database. Red represents tumor samples, while green represents normal tissue samples. (C) BMAL1 and (D) CLOCK expression in GBM and LGG tumor and normal tissues. The red box represents tumor samples, while the grey box represents normal tissue samples. T, tumor; N, normal; TPM, transcripts per million; BMAL1, Brain-Muscle Arnt-Like protein 1; CLOCK, Circadian Locomotor Output Cycles Kaput; ACC, Adrenocortical carcinoma; BLCA, Bladder Urothelial Carcinoma; BRCA, Breast invasive carcinoma; CESC, Cervical squamous cell carcinoma and endocervical adenocarcinoma; CHOL, Cholangiocarcinoma; COAD, Colon adenocarcinoma; DLBC, Lymphoid Neoplasm Diffuse Large B-cell Lymphoma; ESCA, Esophageal carcinoma; GBM, Glioblastoma multiforme; HNSC, Head and Neck squamous cell carcinoma; KICH, Kidney Chromophobe; KIRC, Kidney renal clear cell carcinoma; KIRP, Kidney renal papillary cell carcinoma; LAML, Acute Myeloid Leukemia; LGG, Brain Lower Grade Glioma; LIHC, Liver hepatocellular carcinoma; LUAD, Lung adenocarcinoma; LUSC, Lung squamous cell carcinoma; MESO, Mesothelioma; OV, Ovarian serous cystadenocarcinoma; PAAD, Pancreatic adenocarcinoma; PCPG, Pheochromocytoma and Paraganglioma; PRAD, Prostate adenocarcinoma; READ, Rectum adenocarcinoma; SARC, Sarcoma; SKCM, Skin Cutaneous Melanoma; STAD, Stomach adenocarcinoma; TGCT, Testicular Germ Cell Tumors; THCA, Thyroid carcinoma; THYM, Thymoma; UCEC, Uterine Corpus Endometrial Carcinoma; UCS, Uterine Carcinosarcoma; UVM, Uveal Melanoma. 


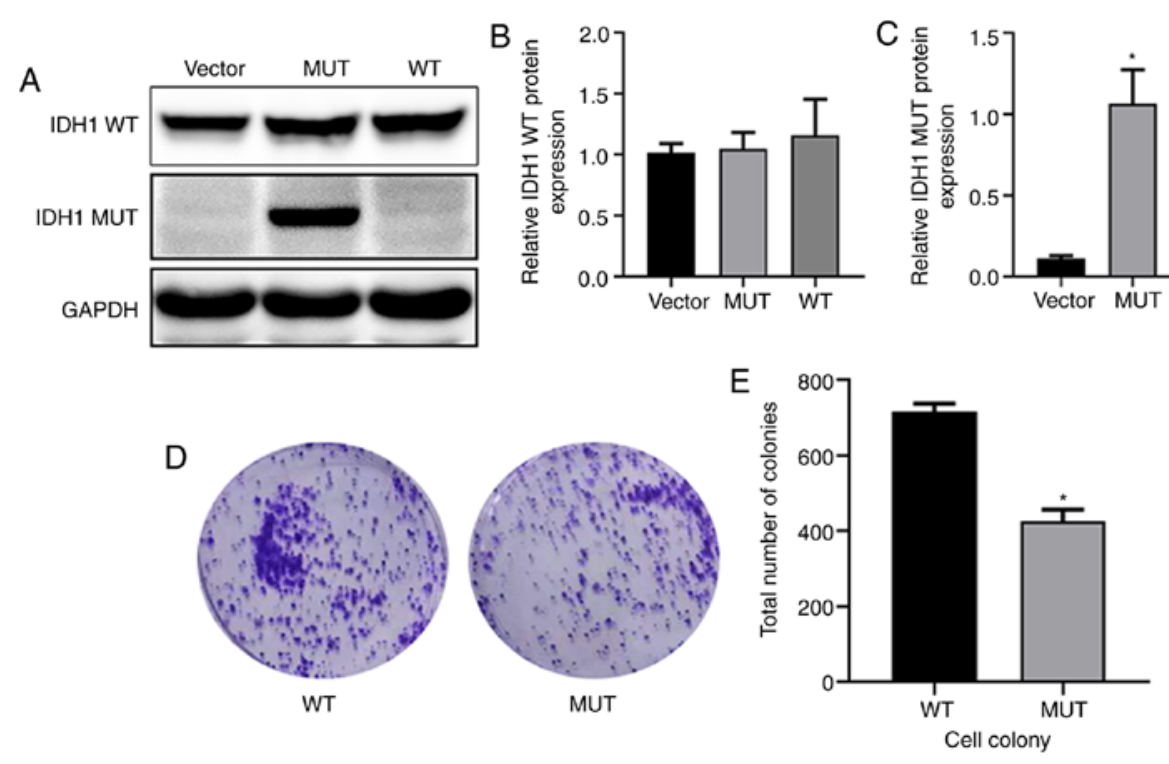

Figure 2. Establishment of a cell model transfected with IDH1 lentivirus, and detection of the IDH1 R132H mutation affects the proliferation of glioma cells. (A) Detection of lentiviral transfection in U87-MG cells. The protein expression levels of IDH1 WT and MUT in each group of U87-MG cells was detected by western blot analysis. (B) Relative IDH1 WT protein expression. (C) Relative IDH1 MUT protein expression. (D and E) Colony formation experiment was used to detect the number of colonies in the two groups. "P<0.05 vs. WT. MUT, mutant; WT, wild-type; IDH1, isocitrate dehydrogenase 1.

normal tissues (Fig. 1C). The expression of BMAL1 in GBM tissue was not significantly different from that in normal tissue (Fig. 1C). CLOCK expression in TGCT tissues was lower than that in normal tissues (Fig. 1B). By contrast, CLOCK expression was higher in diffuse large pancreatic adenocarcinoma (PAAD), stomach adenocarcinoma (STAD) and thymoma (THYM) tissues compared with that in normal tissues (Fig. 1B). Additionally, CLOCK expression in brain LGG was higher than that in normal tissues (Fig. 1D). The expression of CLOCK in GBM tissues was slightly higher compared with that in normal tissues (Fig. 1D). However, how the rhythm genes are expressed in IDH1 R132H mutated glioma is unclear. Therefore, further experiments were performed to investigate this.

Introduction and expression analysis of WT and MUT IDHI genes in U87-MG cells. U87-MG cells were transfected with lentivirus. Western blot analysis (Fig. 2A) indicated that after $72 \mathrm{~h}$ of lentiviral infection, IDH1 WT protein levels in WT U87-MG cells were slightly higher than those in the MUT U87-MG cells (Fig. 2B). The IDH1 MUT protein was highly expressed in the MUT group (Fig. 2C). The effect of IDH1 gene mutation on U87-MG cell proliferation was detected through colony formation experiments, and the results indicated that significantly fewer new colonies were formed in the MUT group than in the WT group (Fig. 2D and E). The current results indicated that a cell model of IDH1 lentivirus transfection was successfully established and that IDH1 R132H mutation influenced the colony formation of glioma cells.

Effects of IDH1 gene mutation on the cell cycle of U87-MG cells. Cell cycle distribution was detected by flow cytometry. In the WT group, $34.41 \%$ of cells were in S phase, while in the MUT group, $19.39 \%$ of cells were in S phase (Fig. 3A-C and G). The proportion of cells in S phase in the MUT group was markedly lower than those in the WT and Vector groups (Fig. 3A-C and G). The proportion of cells in
$\mathrm{G}_{1}$ phase in the MUT group was $72.83 \%$, which was higher than that in the WT group (57.83\%); additionally, there was no marked difference in the proportion of cells in $\mathrm{G}_{2}$ phase among the groups (Fig. 3A-C and G), suggesting that the IDH1 $\mathrm{R} 132 \mathrm{H}$ mutation may inhibit cells from entering $\mathrm{S}$ phase. To further describe the changes in the cell cycle, the expression levels of $\mathrm{S}$ phase-associated proteins (Cyclin A and CDK2), $\mathrm{G}_{2} / \mathrm{M}$ phase-associated protein (Cyclin B1), $\mathrm{G}_{1}$ phase-associated proteins (Cyclin D3 and CDK4), inhibition of early $\mathrm{G}_{1}$ phase P18 protein via inhibiting Cyclin D-CDK4/6 $(44,45)$ and inhibition of late $G_{1}$ phase $P 27$ and $P 21$ proteins via Cyclin E-CDK2 (46) were analyzed by western blot analysis (Fig. 3D). The results revealed that the protein expression levels of Cyclin A and CDK2 were significantly lower in the MUT group than in the WT and vector groups (Fig. 3E and F). There were no significant differences in CyclinB1 expression among the groups (Fig. 3H). The expression levels of Cyclin D3 and CDK4 were significantly higher in the MUT group (Fig. 3I and J). The protein expression levels of P18 protein in the MUT group were significantly lower than in the WT group, and the expression levels of $\mathrm{P} 27$ and $\mathrm{P} 21$ protein in the MUT group were significantly higher than those in the WT and vector groups (Fig. 3K-M). Therefore, the flow cytometry results were verified by detecting the protein expression levels of relevant cyclins, indicating that the IDH1 R132H mutation may affect the regulation of the cell cycle.

Effect of IDH1 gene mutation on biological rhythm genes in U87-MG cells. Abnormal clock gene expression and circadian rhythm disorders are closely associated with the occurrence and development of tumors (47). Western blot analysis revealed that the expression levels of the biological rhythm genes BMAL1 and CLOCK in the IDH1 MUT group were significantly lower than those in the WT group (Fig. 4A-C). Transcriptionally, the clock is driven by positive regulators of the loop. Basic helix-loop-helix heterodimeric transcription 

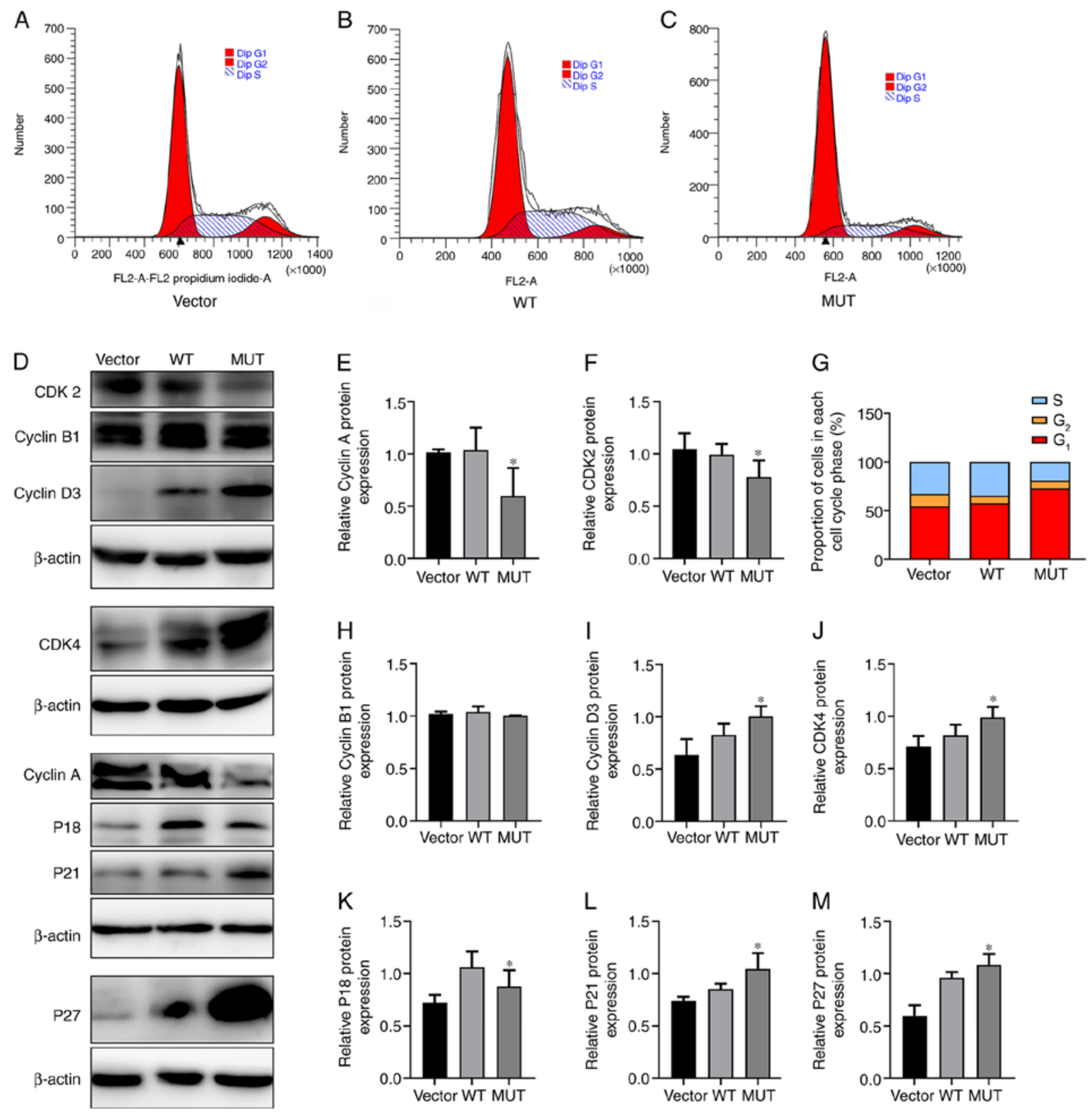

Figure 3. Effect of IDH1 gene mutation on cell cycle. Changes in (A) vector, (B) WT and (C) MUT U87-MG cells after transfection with lentivirus. The cell cycle changes in each group were detected by flow cytometry. (D) Protein expression levels of CyclinA, CyclinB1, CyclinD3, CDK2, CDK4, P18, P21 and P27 in each group of U87-MG cells were detected by western blot analysis. Quantification of relative protein expression levels of (E) CyclinA and (F) CDK2. (G) Proportion of cells in each cell cycle phase in each group. Semi-quantification of relative protein expression levels of (H) CyclinB1, (I) CyclinD3, (J) CDK4, (K) P18, (L) P21 and (M) P27. "P<0.05 vs. WT. MUT, mutant; WT, wild-type; IDH1, isocitrate dehydrogenase 1.

factors (CLOCK/BMAL1 or BMAL1/NPAS2) regulate the expression levels of key circadian genes, including CRY genes (Cry1 and Cry2) and PER genes (PER1, PER2, and PER3), which are negative regulators of the circadian loop $(48,49)$. CRY and PER form a transcriptional repressor complex that enters the nucleus to repress CLOCK/BMAL1 activity, thus creating a negative feedback loop to control the clock (50). Therefore, the expression levels of negative regulators of clock genes were also examined. The negative regulatory factors of biological rhythm PER1, PER2, PER3, Cry1 and Cry2 were significantly downregulated compared with the WT group (Fig. 4D-I). These results indicated that the IDH1 R132H mutation may change the expression levels of biological rhythm genes and may participate in the regulation of the cell biological rhythm.
Effects of IDH1 gene mutation on Smad signaling molecules. IDH1 regulates the TGF- $\beta$ signaling feedback loop through $\alpha$-ketoglutarate $(\alpha-\mathrm{KG})$ and TGF- $\beta$ R)-IDH1-Canine adenovirus 1 (Cav1) to enhance TGF- $\beta$ signaling in a regulatory network between cellular signaling and cell metabolism (51). Additionally, TGF- $\beta$ is an important regulator of the physiological clock $(52,53)$. Therefore, the present study further investigated any changes in the TGF- $\beta /$ Smad signaling pathway in IDH1 R132H mutated glioma cells. Western blot assays were used to detect the effects of MUT and WT IDH1 on the levels of p-Smad2, p-Smad3, Smad2, Smad2-3, Smad3 and Smad4 in U87-MG cells (Fig. 5A). Compared with the WT group, the MUT group exhibited significantly decreased protein expression levels of Smad2, Smad3 and Smad2-3 

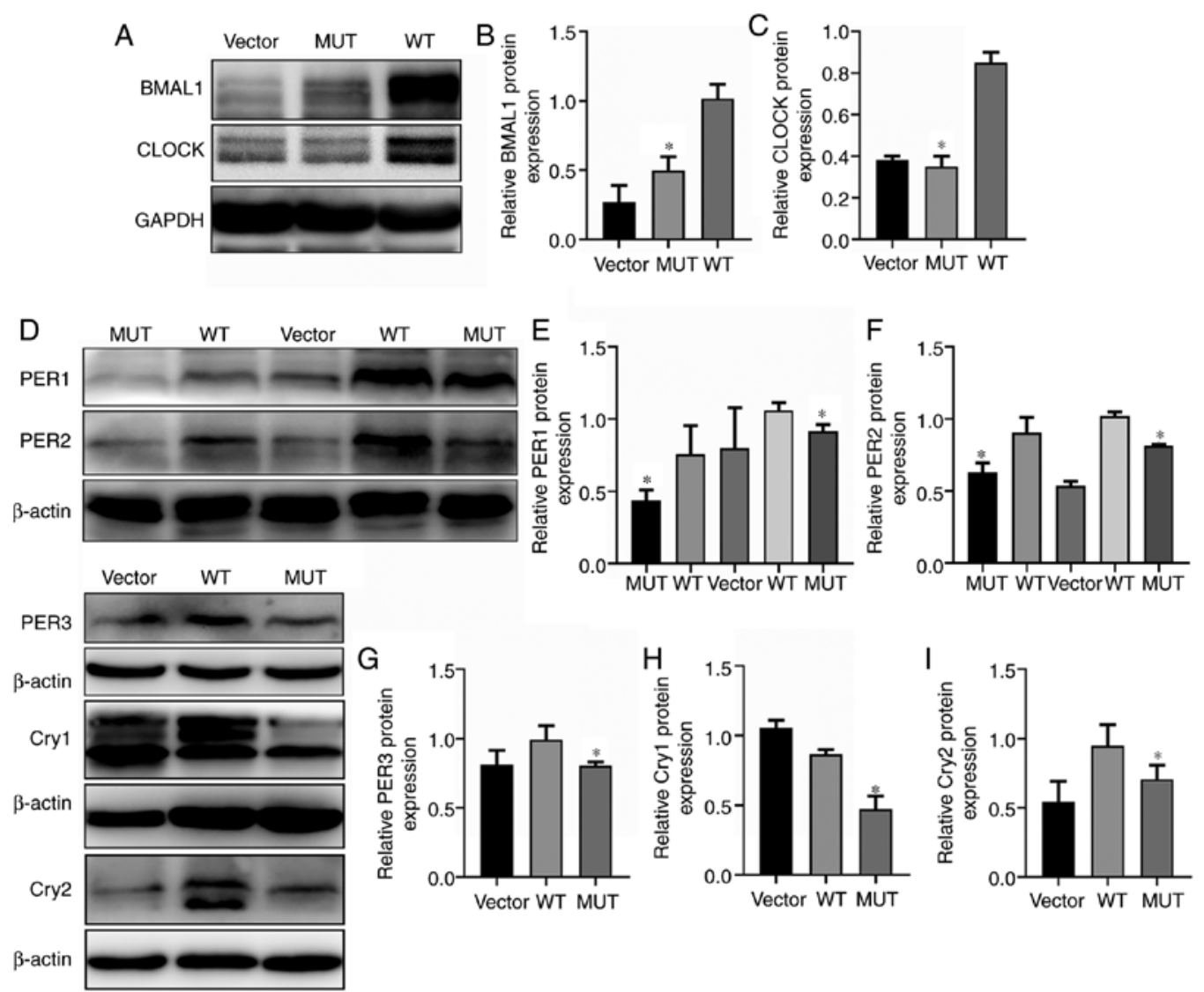

Figure 4. Effects of IDH1 gene mutation on biological rhythm genes. (A) Protein expression levels of BMAL1 and CLOCK in the WT, MUT and vector groups. Quantification of relative protein expression levels of (B) BMAL1 and (C) CLOCK. (D) Protein expression levels of PER1, PER2, PER3, CRY1 and CRY2 in the WT, MUT and vector groups. Quantification of relative protein expression levels of (E) PER1, (F) PER2, (G) PER3, (H) Cry1 and (I) Cry2. "P<0.05 vs. WT. MUT, mutant; WT, wild-type; IDH1, isocitrate dehydrogenase 1; BMAL1, Brain-Muscle Arnt-Like protein 1; CLOCK, Circadian Locomotor Output Cycles Kaput; PER, Period; Cry, Cryptochrom.

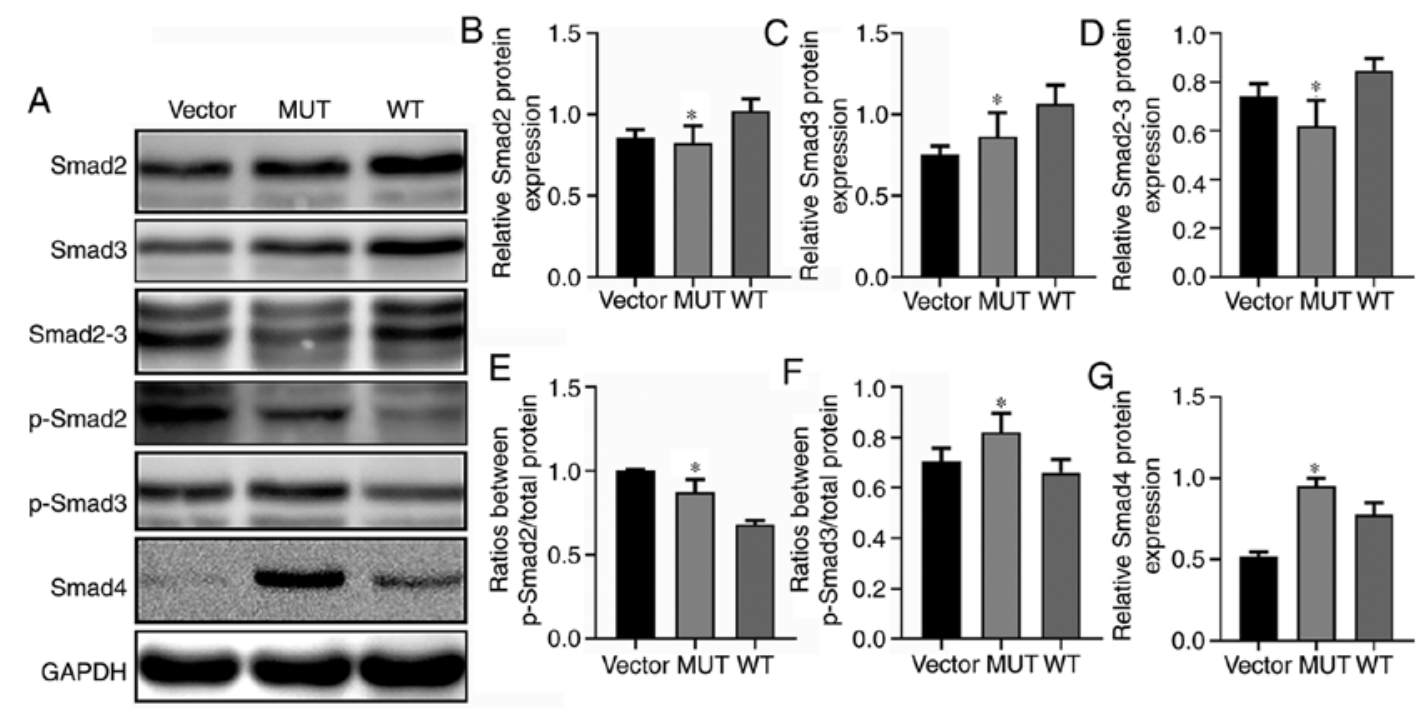

Figure 5. Changes in TGF- $\beta /$ Smad signaling pathway-associated proteins in the IDH1 WT and MUT groups. (A) Expression levels of proteins associated with the Smad signaling pathway were detected by western blot analysis. Quantification of relative protein expression levels of (B) Smad2, (C) Smad3, (D) Smad2-3, (E) p-Smad2/total protein, (F) p-Smad3/total protein and (G) Smad4. "P<0.05 vs. WT. MUT, mutant; WT, wild-type; IDH1, isocitrate dehydrogenase 1; p, phosphorylated.

(Fig. 5B-D) and significantly increased levels of p-Smad2 and p-Smad3 (Fig. 5E and F). Furthermore, Smad4 expression was significantly upregulated in the MUT group compared with in the WT group (Fig. 5G). TGF- $\beta$ initiates signaling pathways by phosphorylating Smad2 and Smad3, and phosphorylated Smad2 and Smad3 bind to Smad4 to form complexes that 
are transferred to the nucleus to interact with multiple transcription factors in order to induce cellular responses $(54,55)$. Thus, it was hypothesized that the IDH1 R132H mutation may affect biological rhythm genes of glioma cells through the TGF- $\beta$ /Smad signaling pathway.

\section{Discussion}

Glioma arises from glial cells and most often occurs in the brain (56). Glioma accounts for $\sim 30 \%$ of central nervous system tumors and $80 \%$ of malignant brain tumors (57). It is characterized with highly infiltrative growth and a poor prognosis. The most common genetic changes in glioma include IDH1/2 mutations, TP53 mutations and 1p/19q heterozygous deletion, which are present in most cases $(>90 \%)$ of glioma $(58,59)$. The most common and early genetic change in glioma is the IDH1/2 mutation. The most common IDH1 mutation results in the amino acid substitution of R132, which is located at the active site of the enzyme (60). IDH1 mutated glioma cells have a reduced proliferative rate (61). Numerous diseases follow circadian rhythms, and there is growing evidence that circadian disruption may be a risk factor for cancer in humans (62). A previous study has reported that the BMAL1 gene serves a role as a potential tumor suppressor gene in pancreatic cancer by activating the P53 tumor suppressor signaling pathway (63). Circadian rhythm genes activate certain pathways in tumors or are activated by certain pathways to affect the development of tumors. $\beta$-catenin showed an increased expression in NIH-3T3 cells after BMAL1 overexpression, indicating that activation of the canonical Wnt pathway may be the mechanism underlying the effect of the circadian clock gene BMAL on promoting cell proliferation (64). BMAL1 suppresses cancer cell invasion by blocking the PI3K-Akt-MMP-2 signaling pathway (65).

However, to the best of our knowledge, there are no studies on the mechanism between IDH1 R132H mutation and biological rhythm genes. Therefore, the present study aimed to describe the role of biological rhythm genes in glioma cells with IDH1 R132H mutation to determine how the IDH1 R132H mutation affects biological rhythm genes and the proliferation of glioma cells.

The current study revealed that the IDH1 R132H mutation in U87-MG cells decreased the number of new colonies formed by tumor cells. The effect of the IDH1 R132H mutation on the cell cycle was further investigated, leading to increased cells in $G_{1}$ phase and decreased cells in $S$ phase. The effect of the mutation on cyclins was also examined. The expression levels of various cyclically related proteins was altered, including the $\mathrm{S}$ phase-associated proteins Cyclin $\mathrm{A}$ and CDK2. No change in Cyclin B1 expression in $G_{2} / M$ phase was observed. The expression levels of the $G_{1}$ phase-associated proteins Cyclin D3 and CDK4 were increased in the MUT group compared with in the WT group. The protein expression levels of P18 were decreased, while those of P21 and P27 were increased in the MUT group compared with in the WT group. Subsequently, the effect of the IDH1 R132H mutation was examined on biological rhythm genes. To the best of our knowledge, the current study was the first to analyze the effect of the IDH1 R132H mutation on biological rhythm. The IDH1 $\mathrm{R} 132 \mathrm{H}$ mutation significantly decreased the protein expression levels of both BMAL1 and CLOCK compared with in the WT group, as well as the expression levels of the negative regulation factors PER1, PER2, PER3, Cry1 and Cry2. A previous study has demonstrated that the disturbance of the circadian clock has a strong influence on tumor transformation and growth by affecting various cancer regulatory signaling pathways, including cell cycle, apoptosis and metabolism (40). The detection of biological rhythm genes in the present study revealed that IDH1 R132H mutation inhibited the protein expression levels of CLOCK and BMAL1, and further inhibited PER and CRY proteins. Therefore, it was shown that the IDH1 R132H mutation has an effect on the expression of biological rhythm genes and has been confirmed in in vitro experiments.

Furthermore, how the IDH1 R132H mutation may affect biological rhythm genes was further investigated. Previous studies have shown that the TGF- $\beta$ signaling pathway is closely associated with the occurrence of glioma $(66,67)$. TGF- $\beta$ is a factor that strongly inhibits the proliferation of epithelial, astrocyte and immune cells, and is considered a tumor suppressor (68). Some tumors acquire mutations in components of the TGF- $\beta$ signaling pathway to evade the TGF- $\beta$ cellular inhibitory response (69). On the other hand, in some malignant tumors, including glioma, the ability of TGF- $\beta$ to inhibit cell proliferation and maintain the integrity of the TGF- $\beta$ signaling pathway is selectively lost (70). TGF- $\beta$ signaling decreases differences in IDH1 expression by normalizing the Smad signaling pathway, and inhibition of Cav1 expression by IDH1 is regulated by $\alpha-\mathrm{KG}$ epigenetic regulation. Finally, downregulation of Cav1 expression interrupts the degradation of TGFBR and enhances the Smad signal. IDH1 regulates the TGF- $\beta$ signaling feedback loop through $\alpha-\mathrm{KG}$ and TGFBR-IDH1-Cav1 to enhance TGF- $\beta$ signaling in a regulatory network between cellular signaling and cell metabolism (51). In the present study, it was observed that the IDH1 R132H mutation significantly decreased the protein expression levels of Smad2, Smad3 and Smad2-3, and significantly upregulated the levels of $\mathrm{p}-\mathrm{Smad} 2, \mathrm{p}-\mathrm{Smad} 3$ and Smad4. A previous study has suggested that TGF- $\beta$ is a multipotent cytokine that controls tissue homeostasis and embryonic development. TGF- $\beta$ binds and activates a membrane receptor serine threonine kinase complex to phosphorylate Smad2 and Smad3; after phosphorylation, Smad proteins accumulate in the nucleus and form complexes with transcription factors, such as Smad4, to regulate transcription (54). The present study revealed that the IDH1 R132H mutation affected the TGF- $\beta$ /Smad signaling pathway. Additionally, TGF- $\beta$ is an important regulator of the physiological clock. A previous study has demonstrated that TGF- $\beta$, by regulating the expression of positive and negative regulators of circadian rhythm oscillation, serves a vital role in regulating circadian rhythm. Adenovirus-mediated TGF- $\beta$ expression can significantly induce BMAL1 and NPAS2 expression (71). It has been demonstrated that the expression levels of the TGF- $\beta$-activated transcription factor Smad3 display similar expression patterns with BMAL1, and that Smad3 functions as an upstream molecule of BMAL1, explaining how TGF- $\beta$ induces BMAL1 expression (53). However, TGF- $\beta$ strongly inhibits the expression levels of PER1, PER2, PER3, Rev-erb $\alpha$, retinoic acid receptor-related orphan receptor $\alpha$ and D-site albumin promoter-binding protein (71). Therefore, increased expression levels of TGF- $\beta$ and negative regulators of the circadian 
clock prolong the arousal cycle (52). The impact of CLOCK and BMAL1 on cancer pathogenesis is highly context- and disease-dependent (50). For instance, CLOCK or BMAL1 provide tumor suppressor-like functions in prostate, breast, ovarian and pancreatic cancer, but exhibit tumor-promoting roles in colorectal cancer and acute myeloid leukemia $(50,72)$. In glioma, CLOCK or BMAL1 are tumor-promoting factors that regulate glioma cell proliferation and migration via regulating the $\mathrm{NF}-\kappa \mathrm{B}$ signaling pathway (73), and can support glioma stem cell function via regulation of anabolic metabolism (74). Therefore, the current study hypothesized that the IDH1 R132H mutation may affect biological rhythm genes through the TGF- $\beta /$ Smad signaling pathway, thus affecting the proliferation of glioma cells.

In summary, the IDH1 gene serves crucial roles in the occurrence and development of numerous types of tumor (75), and imbalance in the circadian clock plays an important role in the development of malignant tumors, including glioma (76). The present study revealed that the IDH1 R132H mutation affected the expression levels of cyclin and biological rhythm genes, and thus may affect the occurrence of glioma. Based on the current results, it was hypothesized that IDH1 mutation may affect the expression levels of biological rhythm genes of cells through the TGF- $\beta /$ Smad signaling pathway. To the best of our knowledge, the present results are the first to report the association between IDH1 R132H mutation and biological rhythm genes, as well as the effect on glioma cell proliferation and the possible underlying mechanisms. However, further studies are required to confirm the current results.

\section{Acknowledgements}

Not applicable.

\section{Funding}

The present study was funded by the National Natural Science Foundation of China (grant no. 81560501), the Ningxia Natural Science Foundation (grant no. 2020AAC03351) and the Ningxia Innovation Team of the foundation and clinical researches of diabetes and its complications (grant no. NXKJT2019010).

\section{Availability of data and materials}

The datasets used and/or analyzed during the current study are available from the corresponding author on reasonable request.

\section{Authors' contributions}

$\mathrm{XC}$ and $\mathrm{SH}$ designed the experiments, guided the study and revised the draft. YW performed the experiments and wrote the manuscript with support from all the other authors. YG and NZ performed the data analysis and contributed to the writing of the manuscript. HY, JY and JM participated in some of the experiments and in data collection. FW and $\mathrm{HX}$ participated in some of the data analyses. XC, YW and YG confirmed the authenticity of the raw data. All authors read and approved the final manuscript.

\section{Ethics approval and consent to participate}

Not applicable.

\section{Patient consent for publication}

Not applicable.

\section{Competing interests}

The authors declare that they have no competing interests.

\section{References}

1. Sjoblom T, Jones S, Wood LD, Parsons DW, Lin J, Barber TD, Mandelker D, Leary RJ, Ptak J, Silliman N, et al: The consensus coding sequences of human breast and colorectal cancers. Science 314: 268-274, 2006.

2. Mardis ER,Ding L,Dooling DJ,Larson DE,McLellan MD,Chen K, Koboldt DC, Fulton RS, Delehaunty KD, McGrath SD, et al: Recurring mutations found by sequencing an acute myeloid leukemia genome. N Engl J Med 361: 1058-1066, 2009.

3. Cardaci S and Ciriolo MR: TCA cycle defects and cancer: When metabolism tunes redox state. Int J Cell Biol 2012: 161837, 2012.

4. Pastore M, Lori G, Gentilini A, Taddei ML, Di Maira G, Campani C, Recalcati S, Invernizzi P, Marra F and Raggi C: Multifaceted aspects of metabolic plasticity in human cholangiocarcinoma: An overview of current perspectives. Cells 9: 596, 2020.

5. Bosnyák E, Michelhaugh SK, Klinger NV, Kamson DO, Barger GR, Mittal S and Juhász C: Prognostic molecular and imaging biomarkers in primary glioblastoma. Clin Nucl Med 42: 341-347, 2017.

6. Krell D, Assoku M, Galloway M, Mulholland P, Tomlinson I and Bardella C: Screen for IDH1, IDH2, IDH3, D2HGDH and L2HGDH mutations in glioblastoma. PLoS One 6: e19868, 2011.

7. Yang H, Ye D, Guan KL and Xiong Y: IDH1 and IDH2 mutations in tumorigenesis: Mechanistic insights and clinical perspectives. Clin Cancer Res 18: 5562-5571, 2012.

8. Zhang Y, Lv W, Li Q, Wang Q, Ru Y, Xiong X, Yan F, Pan T, Lin W and Li X: IDH2 compensates for IDH1 mutation to maintain cell survival under hypoxic conditions in IDH1-mutant tumor cells. Mol Med Rep 20: 1893-1900, 2019.

9. Chittaranjan S, Chan S, Yang C, Yang KC, Chen V, Moradian A, Firme M, Song J, Go NE, Blough MD, et al: Mutations in CIC and IDH1 cooperatively regulate 2-hydroxyglutarate levels and cell clonogenicity. Oncotarget 5: 7960-7979, 2014.

10. Hersh DS, Peng S, Dancy JG, Galisteo R, Eschbacher JM, Castellani RJ, Heath JE, Legesse T, Kim AJ, Woodworth GF, et al: Differential expression of the TWEAK receptor Fn14 in IDH1 wild-type and mutant gliomas. J Neurooncol 138: 241-250, 2018.

11. Sakai Y, Yang C, Kihira S, Tsankova N, Khan F, Hormigo A, Lai A, Cloughesy T and Nael K: MRI radiomic features to predict IDH1 mutation status in gliomas: A machine learning approach using gradient tree boosting. Int J Mol Sci 21: 8004, 2020.

12. Kim W and Liau LM: IDH mutations in human glioma. Neurosurg Clin N Am 23: 471-480, 2012.

13. Ducray F, El Hallani S and Idbaih A: Diagnostic and prognostic markers in gliomas. Curr Opin Oncol 21: 537-542, 2009.

14. Bergo E, Lombardi G, Pambuku A, Della Puppa A, Bellu L, D'Avella D and Zagonel V: Cognitive rehabilitation in patients with gliomas and other brain tumors: State of the Art. Biomed Res Int 2016: 3041824, 2016.

15. Lu C, Ward PS, Kapoor GS, Rohle D, Turcan S, Abdel-Wahab O, Edwards CR, Khanin R, Figueroa ME, Melnick A, et al: IDH mutation impairs histone demethylation and results in a block to cell differentiation. Nature 483: 474-478, 2012.

16. Chen Z, Liu P, Li C, Luo Y, Chen I, Liang W, Chen X, Feng Y, $\mathrm{Xia} \mathrm{H}$ and Wang F: Deregulated expression of the clock genes in gliomas. Technol Cancer Res Treat 12: 91-97, 2013.

17. Luo Y, Wang F, Chen LA, Chen XW, Chen ZJ, Liu PF, li FF, Li CY and Liang W: Deregulated expression of cry1 and cry2 in human gliomas. Asian Pac J Cancer Prev 13: 5725-5728, 2012. 
18. Chang WH and Lai AG: Timing gone awry: Distinct tumour suppressive and oncogenic roles of the circadian clock and crosstalk with hypoxia signalling in diverse malignancies. J Transl Med 17: 132, 2019

19. Chan AB, Huber AL and Lamia KA: Cryptochromes modulate E2F family transcription factors. Sci Rep 10: 4077, 2020

20. Young MW and Kay SA: Time zones: A comparative genetics of circadian clocks. Nat Rev Genet 2: 702-715, 2001.

21. Lowrey PL and Takahashi JS: Genetics of the mammalian circadian system: Photic entrainment, circadian pacemaker mechanisms, and posttranslational regulation. Ann Rev Genet 34 $533-562,2000$

22. Dunlap JC: Molecular bases for circadian clocks. Cell 96 271-290, 1999.

23. Kume K, Zylka MJ, Sriram S, Shearman LP, Weaver DR, Jin X, Maywood ES, Hastings MH and Reppert SM: mCRY1 and mCRY2 are essential components of the negative limb of the circadian clock feedback loop. Cell 98: 193-205, 1999.

24. van der Horst GT, Muijtjens M, Kobayashi K, Takano R, Kanno S, Takao M, de Wit J, Verkerk A, Eker AP, van Leenen D, et al: Mammalian Cry1 and Cry2 are essential for maintenance of circadian rhythms. Nature 398: 627-630, 1999.

25. Vitaterna MH, Selby CP, Todo T, Niwa H, Thompson $C$, Fruechte EM, Hitomi K, Thresher RJ, Ishikawa T, Miyazaki J, et al: Differential regulation of mammalian period genes and circadian rhythmicity by cryptochromes 1 and 2 . Proc Natl Acad Sci USA 96: 12114-12119, 1999.

26. Dibner C, Schibler U and Albrecht U: The mammalian circadian timing system: Organization and coordination of central and peripheral clocks. Ann Rev Physiol 72: 517-549, 2010.

27. Harmer SL, Panda S and Kay SA: Molecular bases of circadian rhythms. Ann Rev Cell Dev Biol 17: 215-253, 2001.

28. Oster H: The genetic basis of circadian behavior. Genes Brain Behav 5 (Suppl 2): S73-S79, 2006.

29. Balsalobre A, Brown SA, Marcacci L, Tronche F, Kellendonk C, Reichardt HM, Schütz G and Schibler U: Resetting of circadian time in peripheral tissues by glucocorticoid signaling. Science 289: 2344-2347, 2000.

30. Damiola F, Le Minh N, Preitner N, Kornmann B, Fleury-Olela F and Schibler U: Restricted feeding uncouples circadian oscillators in peripheral tissues from the central pacemaker in the suprachiasmatic nucleus. Genes Dev 14: 2950-2961, 2000.

31. Yagita K, Tamanini F, van Der Horst GT and Okamura $H$ : Molecular mechanisms of the biological clock in cultured fibroblasts. Science 292: 278-281, 2001.

32. Shearman LP, Sriram S, Weaver DR, Maywood ES, Chaves I, Zheng B, Kume K, Lee CC, van der Horst GT, Hastings MH and Reppert SM: Interacting molecular loops in the mammalian circadian clock. Science 288: 1013-1019, 2000.

33. Mazzoccoli G, Vinciguerra M, Papa G and Piepoli A: Circadian clock circuitry in colorectal cancer. World J Gastroenterol 20 : 4197-4207, 2014

34. Blakeman V, Williams JL, Meng QJ and Streuli CH: Circadian clocks and breast cancer. Br Cancer Res 18: 89, 2016.

35. Cao Q, Gery S, Dashti A, Yin D, Zhou Y, Gu J and Koeffler HP. A role for the clock gene per1 in prostate cancer. Cancer Res 69 7619-7625, 2009

36. Oda A, Katayose Y, Yabuuchi S, Yamamoto K, Mizuma M, Shirasou S, Onogawa T, Ohtsuka H, Yoshida H, Hayashi H, et al: Clock gene mouse period 2 overexpression inhibits growth of human pancreatic cancer cells and has synergistic effect with cisplatin. Anticancer Res 29: 1201-1209, 2009.

37. Zhang S, Zhang J, Deng Z, Liu H, Mao W, Jiang F, Xia Z and Li JD: Circadian clock components ROR $\alpha$ and Bmall mediate the anti-proliferative effect of MLN4924 in osteosarcoma cells. Oncotarget 7: 66087-66099, 2016.

38. Papagiannakopoulos T, Bauer MR, Davidson SM, Heimann M, Subbaraj L, Bhutkar A, Bartlebaugh J, Vander Heiden MG and Jacks T: Circadian rhythm disruption promotes lung tumorigenesis. Cell Metab 24: 324-331, 2016.

39. Yang MY, Lin PM, Hsiao HH, Hsu JF, Lin HY, Hsu CM, Chen IY, Su SW, Liu YC and Lin SF: Up-regulation of PER3 expression is correlated with better clinical outcome in acute leukemia. Anticancer Res 35: 6615-6622, 2015.

40. Farshadi E, van der Horst GTJ and Chaves I: Molecular links between the circadian clock and the cell cycle. J Mol Biol 432: 3515-3524, 2020.

41. Scheving LE, Tsai TH and Scheving LA: Chronobiology of the intestinal tract of the mouse. Am J Anatomy 168: 433-465, 1983.
42. Farshadi E, Yan J, Leclere P, Goldbeter A, Chaves I and van der Horst GTJ: The positive circadian regulators CLOCK and BMAL1 control $\mathrm{G}_{2} / \mathrm{M}$ cell cycle transition through Cyclin B1. Cell Cycle 18: 16-33, 2019.

43. Shan DZ, Zhang C and Cao XM: Preparation and expression of isocitrate dehydrogenase 1 and mutant recombinant lentivirus. Chin J Neuroanatomy 36: 200-206, 2020.

44. Yu H, Yuan Y, Shen H and Cheng T: Hematopoietic stem cell exhaustion impacted by p18 INK4C and p21 Cip1/Waf1 in opposite manners. Blood 107: 1200-1206, 2006.

45. Yuan Y, Shen H, Franklin DS, Scadden DT and Cheng T: In vivo self-renewing divisions of haematopoietic stem cells are increased in the absence of the early $\mathrm{G}_{1}$-phase inhibitor, p18INK4C. Nat Cell Biol 6: 436-442, 2004.

46. Sherr CJ: Cancer cell cycles. Science 274: 1672-1677, 1996.

47. Mandal AS, Biswas N, Karim KM, Guha A, Chatterjee S, Behera $\mathrm{M}$ and Kuotsu K: Drug delivery system based on chronobiology-A review. J Control Release 147: 314-325, 2010.

48. Zhao Q, Zheng G, Yang K, Ao YR, Su XL, Li Y and Lv XQ: The clock gene PER1 plays an important role in regulating the clock gene network in human oral squamous cell carcinoma cells. Oncotarget 7: 70290-70302, 2016.

49. Qu M, Duffy T, Hirota T and Kay SA: Nuclear receptor HNF4A transrepresses CLOCK:BMAL1 and modulates tissue-specific circadian networks. Proc Natl Acad Sci USA 115: E12305-E12312, 2018.

50. Shafi AA and Knudsen KE: Cancer and the circadian clock. Cancer Res 79: 3806-3814, 2019.

51. Hou X, Zhang J, Wang Y, Xiong W and Mi J: TGFBR-IDH1-Cav1 axis promotes TGF-beta signalling in cancer-associated fibroblast. Oncotarget 8: 83962-83974, 2017.

52. Lopez M, Meier D, Muller A, Franken P, Fujita J and Fontana A: Tumor necrosis factor and transforming growth factor beta regulate clock genes by controlling the expression of the cold inducible RNA-binding protein (CIRBP). J Biol Chem 289: 2736-2744, 2014.

53. Sato F, Sato H, Jin D, Bhawal UK, Wu Y, Noshiro M, Kawamoto T, Fujimoto K, Seino H, Morohashi S, et al: Smad3 and Snail show circadian expression in human gingival fibroblasts, human mesenchymal stem cell, and in mouse liver. Biochem Biophys Res Commun 419: 441-446, 2012.

54. Kang Y, He W, Tulley S, Gupta GP, Serganova I, Chen CR, Manova-Todorova K, Blasberg R, Gerald WL and Massagué J: Breast cancer bone metastasis mediated by the Smad tumor suppressor pathway. Proc Natl Acad Sci USA 102: 13909-13914, 2005.

55. Dou C, Lee J, Liu B, Liu F, Massague J, Xuan S and Lai E: BF-1 interferes with transforming growth factor beta signaling by associating with Smad partners. Mol Cell Biol 20: 6201-6211, 2000.

56. Parisot S, Wells W III, Chemouny S, Duffau H and Paragios N: Concurrent tumor segmentation and registration with uncertainty-based sparse non-uniform graphs. Med Image Anal 18 647-659, 2014

57. de Robles P, Fiest KM, Frolkis AD, Pringsheim T, Atta C St Germaine-Smith C, Day L, Lam D and Jette N: The worldwide incidence and prevalence of primary brain tumors: A systematic review and meta-analysis. Neurooncology 17: 776-783, 2015.

58. Eckel-Passow JE, Lachance DH, Molinaro AM, Walsh KM, Decker PA, Sicotte H, Pekmezci M, Rice T, Kosel ML, Smirnov IV, et al: Glioma Groups Based on 1p/19q, IDH, and TERT promoter mutations in tumors. N Engl J Med 372: 2499-2508, 2015.

59. Miller JJ, Shih HA, Andronesi OC and Cahill DP: Isocitrate dehydrogenase-mutant glioma: Evolving clinical and therapeutic implications. Cancer 123: 4535-4546, 2017

60. Ferreira MSV, Sørensen MD, Pusch S, Beier D, Bouillon AS, Kristensen BW, Brümmendorf TH, Beier CP and Beier F: Alternative lengthening of telomeres is the major telomere maintenance mechanism in astrocytoma with isocitrate dehydrogenase 1 mutation. J Neurooncol 147: 1-14, 2020.

61. Karpel-Massler G, Nguyen TTT, Shang E and Siegelin MD: Novel IDH1-targeted glioma therapies. CNS Drugs 33: 1155-1166, 2019

62. Salavaty A, Mohammadi N, Shahmoradi M and Naderi Soorki M: Bioinformatic analysis of circadian expression of oncogenes and tumor suppressor genes. Bioinform Biol Insights 11: 1177932217746991,2017

63. Jiang W, Zhao S, Jiang X, Zhang E, Hu G, Hu B, Zheng P, Xiao J, Lu Z, Lu Y, et al: The circadian clock gene Bmal1 acts as a potential anti-oncogene in pancreatic cancer by activating the p53 tumor suppressor pathway. Cancer Lett 371: 314-325, 2016. 
64. Lin F, Chen Y, Li X, Zhao Q and Tan Z: Over-expression of circadian clock gene Bmal1 affects proliferation and the canonical Wnt pathway in NIH-3T3 cells. Cell Biochem Funct 31: 166-172, 2013.

65. Jung CH, Kim EM, Park JK, Hwang SG, Moon SK, Kim WJ and Um HD: Bmall suppresses cancer cell invasion by blocking the phosphoinositide 3-kinase-Akt-MMP-2 signaling pathway. Oncol Rep 29: 2109-2113, 2013.

66. Guan F, Kang Z, Wang L, Wang K, Mao BB, Peng WC, Zhang BL, Lin ZY, Zhang JT and Hu ZQ: Retinol dehydrogenase 10 promotes metastasis of glioma cells via the transforming growth factor- $\beta$ /SMAD signaling pathway. Chin Med J (Engl) 132: 2430-2437, 2019.

67. Derynck R and Zhang YE: Smad-dependent and Smadindependent pathways in TGF-beta family signalling. Nature 425 : 577-584, 2003.

68. Frei K, Gramatzki D, Tritschler I, Schroeder JJ, Espinoza L, Rushing EJ and Weller M: Transforming growth factor- $\beta$ pathway activity in glioblastoma. Oncotarget 6: 5963-5977, 2015.

69. Huse K, Bakkebø M, Wälchli S, Oksvold MP, Hilden VI, Forfang L, Bredahl ML, Liest $\varnothing 1 \mathrm{~K}$, Alizadeh AA, Smeland EB and Myklebust JH: Role of Smad proteins in resistance to BMP-induced growth inhibition in B-cell lymphoma. PLoS One 7: e46117, 2012.

70. Sadeghi Y, Tabatabaei Irani P, Rafiee L, Tajadini M, Amouheidari A and Javanmard SH: Evaluation of rs1982073 polymorphism of transforming growth factor- $\beta 1$ in glioblastoma. J Res Med Sci 24: 40, 2019.
71. Dong C, Gongora R, Sosulski ML, Luo F and Sanchez CG: Regulation of transforming growth factor-beta1 (TGF- 31 )induced pro-fibrotic activities by circadian clock gene BMAL1. Respir Res 17: 4, 2016.

72. Puram RV, Kowalczyk MS, de Boer CG, Schneider RK, Miller PG, McConkey M, Tothova Z, Tejero H, Heckl D, Järås M, et al: Core circadian clock genes regulate leukemia stem cells in AML. Cell 165: 303-316, 2016.

73. Li A, Lin X, Tan X, Yin B, Han W, Zhao J, Yuan J, Qiang B and Peng X: Circadian gene Clock contributes to cell proliferation and migration of glioma and is directly regulated by tumor-suppressive miR-124. FEBS Lett 587: 2455-2460, 2013.

74. Dong Z, Zhang G, Qu M, Gimple RC, Wu Q, Qiu Z, Prager BC, Wang X, Kim LJY, Morton AR, et al: Targeting glioblastoma stem cells through disruption of the circadian clock. Cancer Discov 9: 1556-1573, 2019.

75. Liu WS, Chan SH, Chang HT, Li GC, Tu YT, Tseng HH, Fu TY, Chang HY, Liou HH, Ger LP and Tsai KW: Isocitrate dehydrogenase 1-snail axis dysfunction significantly correlates with breast cancer prognosis and regulates cell invasion ability. Breast Cancer Res 20: 25, 2018.

76. Xia HC, Niu ZF, Ma H, Cao SZ, Hao SC, Liu ZT and Wang F: Deregulated expression of the Per1 and Per2 in human gliomas. Can J Neurol Sci 37: 365-370, 2010.

This work is licensed under a Creative Commons Attribution-NonCommercial-NoDerivatives 4.0 International (CC BY-NC-ND 4.0) License. 